\title{
TURBO SPECKLE FILTERING APPLIED TO POLSAR DATA
}

\author{
Grégory Farage $^{(1,2)}$, Samuel Foucher ${ }^{(2)}$, Carlos López-Martínez ${ }^{(3)}$, Goze B. Bénié(1) \\ ${ }^{(1)}$ CARTEL, University of Sherbrooke, Sherbrooke, QC, Canada \\ gregory.farage@usherbrooke.ca, gbenie@,usherbooke.ca \\ ${ }^{(2)}$ Computer Research Institute of Montreal, Montreal, QC, Canada \\ sfoucher@crim.ca \\ ${ }^{(3)}$ Remote Sensing Lab. (RSLab.), Signal Theory and Communications Dept. (TSC), \\ Universitat Politecnica de Catalunya (UPC), Campus Nord, Edif. D3-203, Jordi Girona 1-3, 08034 \\ Barcelona, Spain \\ carlos.lopez@tsc.upc.edu
}

\begin{abstract}
Abstract - A new approach for speckle reduction in polarimetric synthetic aperture radar (PolSAR) images based on the turbo iterative principle is proposed. The turbo iterative algorithm shows high performances due to the propagation of the information between two complementary filters. One filter can boost up the results of the other by processing its residue image and retrieving valuable information in the noise subspace.
\end{abstract}

Index Terms - PolSAR, speckle filtering, Turbo principle.

\section{INTRODUCTION}

Recently, Sun et al. [1] proposed a new iterative processing for SAR image restoration based on the turbo iterative principle. The turbo principle has emerged from the turbo codes which are a class of recently-developed highperformance error correction code introduced by Berrou et al. [2]. Such codes use two decoders in an iterative process, so called turbo iterative decoding. This processing results in an error correction approaching the Shannon limit, which is the theoretical limit of maximum information transfer rate over a noisy channel. The concept behind turbo decoding is to pass soft decisions from the output of one decoder to the input of the other decoder, and to iterate this process until they converge to a more reliable solution. The turbo iterative algorithm applied to SAR images [1] shows high performances due to the propagation of the information between two simple filters.

The encouraging results after the turbo iterative principle provide an excellent motivation to investigate a turbo method applied to PolSAR data. Our objective is to carry out an analysis of a PolSAR turbo filter. Here it is quite important to stress on the concept of iteration, where it is not a simple repetition of a filtering process but more like a recurrence of its parameter estimation. Nevertheless, one of the main constraints in such systems is to design two complementary filters that are analogous to the decoders in turbo code. Some filters, e.g., Lee filter [3], tend to obtain a very smooth image while leaving some texture and feature information in the removed noise image that can be called residue image. In contrast, other filters, e.g., wavelet based filter [4], tend to retain the structural information while leaving some noise in the estimated image. Therefore, the interest of turbo iterative algorithm is to make use of the advantages of both filters. One filter can boost up the results of the other by processing its residue image and retrieving valuable information in the noise subspace. In such a case, extrinsic information is exchanged resulting in a better estimation of the polarimetric reflectivity.

In section 2, a background about turbo iterative processing and a description of two elementary filters is provided. In section 3, simulations are carried out to investigate the performances of the presented approach, filtered artificial and real images are shown. Finally, section 4 goes over the main results and runs through further perspectives.

\section{TURBO SPECKLE FILTER}

In reciprocal backscattering case, $S_{h v}=S_{v h}$, polarimetric information can be represented by the scattering vector $\boldsymbol{k}_{S}$,

$$
\mathbf{k}_{S}=\left[\begin{array}{lll}
S_{h h} & \sqrt{2} S_{h v} & S_{v v}
\end{array}\right]^{T}
$$

where $h$ and $v$ represent respectively the transmitting horizontal and receiving vertical linear polarization, and the superscript " $T$ " refers to the matrix transpose. From (1), the polarimetric covariance matrix $\mathbf{C}$ and the span are expressed as follows,

$$
\begin{gathered}
\mathbf{C}=\mathbf{k}_{S} \mathbf{k}_{S}{ }^{* T} \\
\operatorname{span}=\mathbf{k}^{* T} \mathbf{k}=\left|S_{h h}\right|^{2}+2\left|S_{h v}\right|^{2}+\left|S_{v v}\right|^{2}
\end{gathered}
$$

where the superscript "*” refers to the complex conjugate.

$$
\mathbf{I}=\left\{C_{11}, C_{12}, C_{13}, C_{22}, C_{23}, C_{33}\right\}
$$




\subsection{Turbo Iterative Principle}

In the case of a PolSAR filter, the turbo iterative principle consists in generating two component signals, $\mathbf{U}_{1}$ and $\mathbf{U}_{2}$, from the original signal I. As shown in Figure 1. , these two component signals, theoretically uncorrelated, result from the two operators ("interleavers") $\pi_{1}$ and $\pi_{2}$, described by the following equations:

$$
\begin{gathered}
\left.\pi_{1}\right|_{k} \triangleq\left\{\begin{array}{c}
U_{1, k}=\left|\frac{I_{k}}{\psi_{k} \bar{z}_{n, k}}\right|, k=2,3,5 \\
U_{1, k}=\left|\frac{I_{k}}{Z_{21, k}}\right|, k=1,4,6
\end{array}\right. \\
\left.\pi_{2}\right|_{k} \triangleq\left\{\begin{array}{c}
U_{2, k}=\left.\psi_{k} \bar{z}_{n, k} Z_{12, k} e^{j \phi_{k}}\right|_{k=2,3,5} \\
U_{2, k}=\left.Z_{12, k} Z_{21, k}\right|_{k=1,4,6}
\end{array}\right.
\end{gathered}
$$

Where the different parameters are defined as follows:

- $\bar{z}_{n, k}$ refers to the average Hermitian product normalized intensity value of $\mathbf{Z}_{\mathbf{2 1}}$, with $\rho_{k}=\left|\rho_{k}\right| \exp \left(j \phi_{x, k}\right)$ as the complex correlation coefficient of the channel $k$.

$$
\bar{z}_{n, k}=\frac{\pi}{4}{ }_{2} F_{1}\left(-\frac{1}{2},-\frac{1}{2} ; 1 ;\left|\rho_{k}\right|^{2}\right)
$$

- $\psi_{k}$ represents the average power in the two channels of $\mathrm{Z}_{21}$

$$
\psi_{k}=\sqrt{E\left\{\left|S_{p}\right|^{2}\right\} E\left\{\left|S_{q}\right|^{2}\right\}}
$$

$\pi_{1}$ and $\pi_{2}$ are derived from the statistical description of polarimetric SAR data of Tough et al. [5].and from the PolSAR noise model presented. The signal $\mathbf{U}_{1}$ is defined as a multiplicative noise by López-Martínez and Fàbregas [6] and its pdf has been found relatively invariant to change in coherence values as can be seen in Figure 2. The pdf of $\left\{U_{1, k}\right\}_{k=2,3,5}$ obtained from the distribution of the Hermitian product amplitude expressed by Tough et al. [5] is

$$
p_{U}(u)=\frac{4 \bar{z}_{n} u}{1-|\rho|^{2}} I_{0}\left(\frac{2 \bar{z}_{n}|\rho| u}{1-|\rho|^{2}}\right) K_{0}\left(\frac{2 \bar{z}_{n} u}{1-|\rho|^{2}}\right)
$$

where $I_{0}$ is a modified Bessel function of the first kind, $K_{0}$ is a modified Bessel function of the second kind, and $\rho$ is defined as:

$$
\rho=\frac{\left\langle S_{p} S_{q}\right\rangle}{\sqrt{\left\langle\left|S_{p}^{2}\right|\right\rangle\left\langle\left|S_{q}^{2}\right|\right\rangle}}
$$

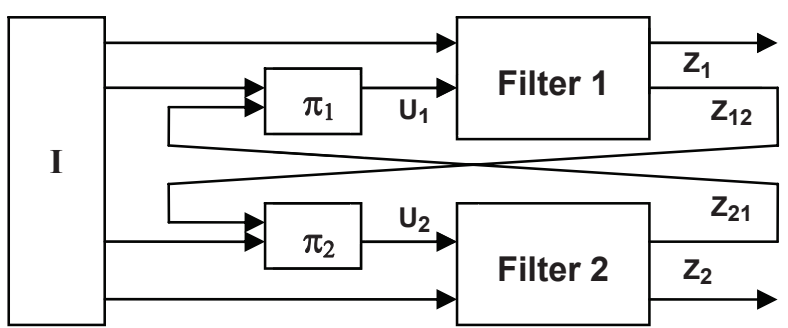

Figure 1. Scheme of Turbo Iterative Principle.
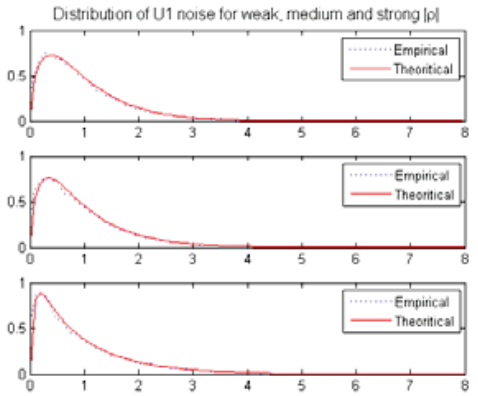

Figure 2. Empircal and Theoritical Probability Density Funcfion of $\mathbf{U}_{1}$.

Next, two different filters have to be chosen in a way that their performances complement each other. Filter 1 should present a tendancy to reduce noise with a good preservation of spatial features, and should process the residue noise to recover valuable information in the noise subspace. In contrast, filter 2 should demonstrate a speckle reduction with good polarimetric parameters estimation. Then, the output signals $\mathbf{Z}_{12}$ and $\mathbf{Z}_{\mathbf{2 1}}$ are the information exchanged between both filters in order to balance the speckle filter performances. At each iteration, $\mathbf{z}_{\mathbf{1 2}}$ and $\mathbf{Z}_{\mathbf{2 1}}$ are estimated to enhance the results of each filter and to compensate their costs. The iterative process stops when the change in $\mathbf{Z}_{\mathbf{2 1}}$ is small.

\subsection{Complementary filters}

\subsubsection{PolSAR Wavelet Shrinkage}

For filter 1, a wavelet shrinkage filter developed by Farage et al. [4] is considered. The wavelet transform employed is based on a Stationary Wavelet Transform (SWT). In addition, the significant coefficient values are enhanced by summing the squared coefficients of all the channels. By squaring real edge coefficients, they tend to become larger for coarser scales, while noise coefficients become smaller. The wavelet shrinkage technique makes it possible to identify significant wavelet coefficients (i.e., mainly those generated by edges and point targets) to recover the information over-filtered by filter 2 and contained in signal $\mathbf{U}_{1}$. The threshold technique is based on percentile method supported by (9).

Therefore, the output of filter 1 relates to some retrieved spatial feature information that is used by filter 2 to improve the estimation of weighting coefficients. 


\subsubsection{Lee filter}

One of the filters experimented for the filter 2 in the design of the turbo algorithm is the Lee filter [3]. It is based on the estimation of the local variance statistics. The filtering weights are determined using the span image that benefits from the scattering characteristics of the $\mathrm{HH}, \mathrm{VV}$ and $\mathrm{HV}$ intensities. The local linear minimum mean-square filter is obtained as such

$$
\hat{x}=\bar{y}+b(y-\bar{y})
$$

where $\hat{x}$ is the filtered pixel value, $\bar{y}$ is the local mean, and $b$ is the weighting function expressed as

$$
b=\frac{\operatorname{var}(x)}{\operatorname{var}(y)} \text { and } \operatorname{var}(x)=\frac{\operatorname{var}(y)-\bar{y}^{2} \sigma_{v}^{2}}{\left(1+\sigma_{v}^{2}\right)}
$$

where $\sigma^{2}{ }_{v}$ is the speckle variance. In the turbo iterative process, the variance $\operatorname{var}(x)$ of the coefficient $b$ is computed from the extrinsic information $\mathbf{U}_{2}$ in (5), and $\operatorname{var}(y)$ is determined from the original data $\mathbf{I}$.

\subsubsection{Model-Based PolSAR filter}

Alternatively, the MB-PolSAR filter [7] can be used for filter 2. The MB-PolSAR filter is based on the multiplicativeadditive speckle noise model for multidimensional SAR data stated as

$$
\begin{aligned}
S_{p} S_{q}^{*}= & \psi_{k} \bar{z}_{n, k} N_{c} n_{m} e^{j \phi_{x, k}}+\psi_{k}\left(\left|\rho_{k}\right|-N_{c} \bar{z}_{n, k}\right) e^{j \phi_{x, k}} \\
& +\psi_{k}\left(n_{a r}+j n_{a i}\right)
\end{aligned}
$$

where $N_{c}$ is expressed as

$$
N_{c}=\frac{\pi}{4}\left|\rho_{k}\right|{ }_{2} F_{1}\left(\frac{1}{2}, \frac{1}{2} ; 2 ;\left|\rho_{k}\right|^{2}\right)
$$

This filter approach is based on processing the elements of the covariance PolSAR data matrix according to the estimation of the complex correlation coefficient. Therefore, in the turbo iterative filter, the estimation processing of $\left|\rho_{\mathrm{k}}\right|$ is conducted by the extrinsic information $\mathbf{U}_{2}$ in (5).

\subsection{Despeckling Method}

The various steps of the full non parametric filtering method are as follows:

1. Use a mask to preserve original point targets values.

2. Set initial reference image so that $\mathbf{Z}_{\mathbf{2 1}}$ is the lowfrequency images of $\mathbf{I}$.

3. Get the residue image $U_{1}$ from the operator $\pi_{1}$.

4. Apply filter $\mathbf{I}$ based on $\mathbf{U}_{\mathbf{1}}$ and $\mathbf{I}$ according to PolSAR wavelet filter [4].

5. Apply filter 2 and estimate $b$ coefficient (Lee filter) or $\left|\rho_{\mathrm{k}}\right|$ (MB-PolSAR filter) from $\mathbf{U}_{2}$ and $\mathbf{I}$.

6. Stop iteration if change in $\mathbf{Z}_{\mathbf{2 1}}$ is small or else go to step 3 and change the reference image into the new $\mathbf{Z}_{\mathbf{2 1}}$.
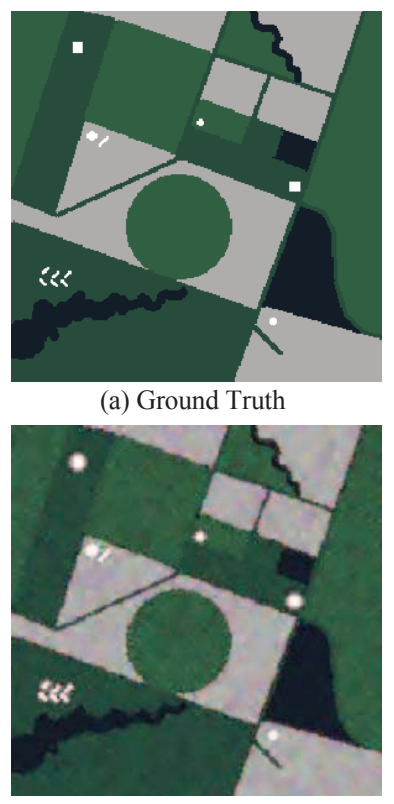

(c) SSC 4 levels, $\mathrm{H}-\alpha=79.5 \%$ $\mathrm{EP}=0.161, \mathrm{ENL}=20.27$

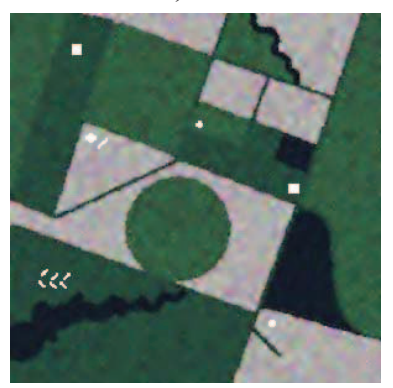

(e) Turbo Lee7x 7 , iter4, Output filter $1, \mathrm{H}-\alpha=81 \%$ $\mathrm{EP}=0.1090, \mathrm{ENL}=26.5$ (a) Ground Truth

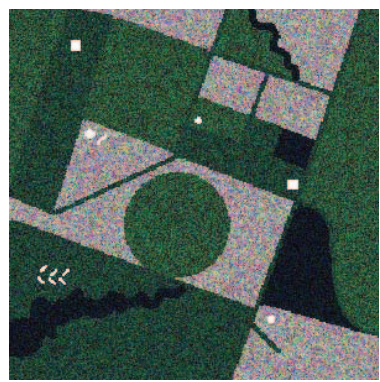

(b) Original Image

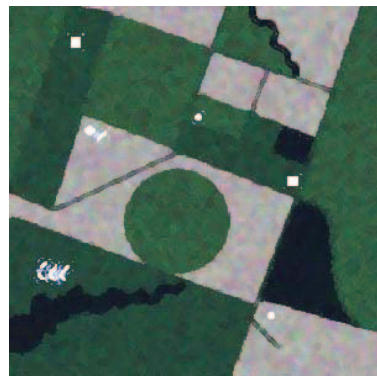

(d) Enhanced Lee $7 \times 7, H-\alpha=76.9 \%$ $\mathrm{EP}=0.260, \mathrm{ENL}=9.65$

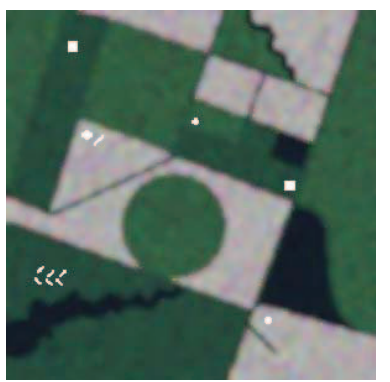

(f) Turbo MB 7x7, iter 4, Output filter $2, \mathrm{H}-\alpha=83 \%$ $\mathrm{EP}=0.216, \mathrm{ENL}=34.1$
Figure 3. Display color, red $=2|\mathrm{Shv}|^{2}$, green $=|\mathrm{Svv}|^{2}$, blue $=|\mathrm{Shh}|^{2} . \quad \mathrm{H}-$ $\alpha$ is Entropy-Alpha classification rate.

\section{RESULTS}

\subsection{Experiments Using Simulated Images}

Speckle reduction performances are evaluated on simulated PolSAR images (single look complex), produced from the Cholesky factorization of typical samples polarimetric responses. Five areas with different scattering properties are selected within an E-SAR image (L-band, DLR, Oberpfaffenhofen area). The final PolSAR image is shown in Figure 3., as well as the ground truth image. We evaluated the performances of the artificial PolSAR image filtering on its three power bands with the following indicators:

- Equivalent number of looks (ENL): a measure that indicates the strength of the noise reduction; we computed the ENL for each power channel and each 
scattering classes (we excluded point targets) and we took the mean value.

$$
E N L=\frac{1}{3 \times 5} \sum_{n=1,4,6} \sum_{l=1, . .5} \frac{<I_{n}>^{2}}{E\left[\left(I_{n}-<I_{n}>\right)^{2} \mid l\right]}
$$

- Edge strength preservation (EP): preservation of the edge magnitudes of filtered image is compared to the ground truth image (the smaller the EP the more significant it is).

$$
E P=\frac{1}{3 \times 5} \sum_{n=1,4,6} \sum_{b=1, \ldots, 5}\left|1-\frac{\sum_{\mathbf{x} \in \text { Edges }}\left\|\nabla I_{n}(\mathbf{x})\right\|}{\sum_{\mathbf{x} \in \text { Edges }}\left\|\nabla I_{n}^{G T}(\mathbf{x})\right\|}\right|
$$

\subsection{ExperimentsUsing PolSAR Images from Airborne} Sensor

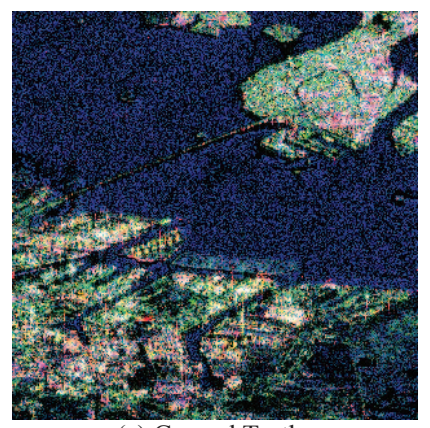

(a) Ground Truth

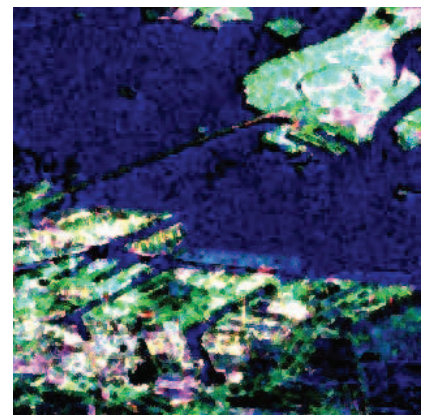

(c) Enhanced Lee $7 \times 7$

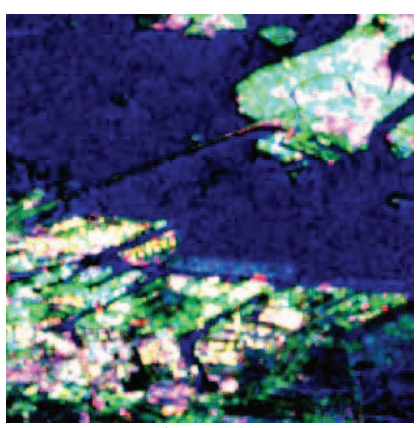

(b) Lee $7 \times 7$

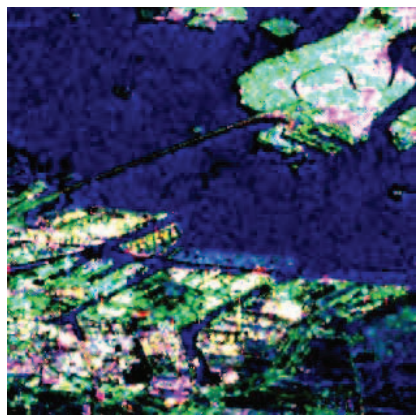

(d) Turbo Lee $7 \times 7$ it. 4output filter 1

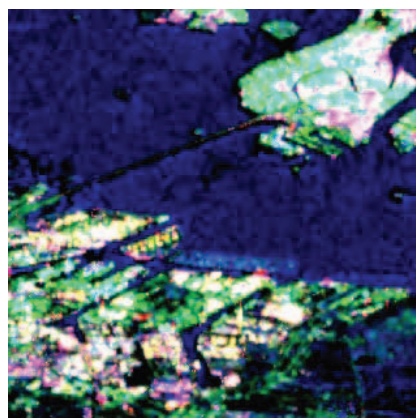

(e) Turbo Lee $7 \times 7$ it. 4 output filter 2
Figure 4. Display color, red $=2|\mathrm{Shv}|^{2}$, green $=|\mathrm{Svv}|^{2}$, blue $=|\mathrm{Shh}|^{2}$.

In case of real images, the filters were applied to the Japan Aerospace Exploration Agency (JAXA) ALOS L-band
POLSAR data of a Venizia, Italy, image. These data are single look complex, and processed by all filters with a window size $7 \times 7$.

\section{DISCUSSION AND CONCLUSION}

It can be seen from the experiment results shown in Figure 3. that Turbo speckle filtering offers higher ENL values with better EP indexes compared to standards filters presented in the experiment (i.e., enhanced Lee filter [8] and SSC wavelet filter [4]). The turbo iterative processing adaption to PolSAR data improves clearly the trade-off between the noise reduction and spatial features preservation. The Entropy-Alpha rate classification has also been enhanced and especially with the turbo-MB filter. In Figure 4. , we can observe that Turbo Lee presents a remarkable capacity to preserve edges and meaningful spatial features. The propagation of information flowing in the turbo filter allows reaching for a more optimum result.

The turbo principle applied to PolSAR data yields encouraging results, and consequently will be the subject of further studies.

\section{ACKNOWLEDGMENT}

This work has been supported in part by the NSERC of Canada (Discovery Grant) and the MDEIE of the "Gouvernement du Québec". The authors wish to thank the DLR (German Aerospace Center) for so graciously providing them with the E-SAR dataset.

\section{REFERENCES}

[1] H. Sun, H. Maitre, and B. Guan, "Turbo image restoration," Proc. ISSPA, Vol. 1, pp. 417-420, July 2003.

[2] C. Berrou, A. Glavieux, and P. Thitimajshima, "Near Shannon-Limit error coding and decoding: turbo codes," Proc. ICC'93, Geneva, 1993.

[3] J. S. Lee, M. R. Grunes, and S. A. Mango, "Speckle reduction in multipolarization and multifrequency SAR imagery," IEEE Trans. Geosci. Remote Sensing, Vol. 29, No 4, pp. 535-544, July 1991.

[4] G. Farage, S. Foucher, and G. Bénié, "PolSAR image filtering based on feature detection using the wavelet transform ," Proc. IGARSS'07, pp. 2648-2652, July 2007.

[5] R. J. A. Tough, D. Blacknell, and S. Quegan, "A statistical description of polarimetric and interferometric synthetic aperture radar data," Proceedings: Mathematical and Physical Sciences, Vol. 449, No. 1937, pp. 567-589, June 1995.

[6] C. López-Martínez, and Fàbregas,"Polarimetric SAR Speckle Noise Model," IEEE Trans. Geosci. Remote Sensing, Vol.41, No. 10, pp. 2232-2242, Oct.2003.

[7] C. López-Martínez, and Fàbregas, "Multidimensional speckle noise reduction in synthetic aperture radar images," Proc. IGARSS'07, pp. 176-179, July 2007.

[8] J.S. Lee, M. R. Grunes, and G. De Grandi, "Polarimetric SAR Speckle Filtering and its Implication for Classification", IEEE Trans. Geosci. Remote Sensing, Vol. 37, No 5, pp 2363-2373, Sep. 1999. 